\title{
Mitigation of shock-induced flow separation using magnetohydrodynamic flow control
}

\author{
R BALASUBRAMANIAN*, K ANANDHANARAYANAN, R KRISHNAMURTHY and \\ DEBASIS CHAKRABORTY
}

\author{
Directorate of Computational Dynamics, Defence Research and Development Laboratory, Hyderabad 500058, \\ India \\ e-mail: bals.cfd@gmail.com
}

MS received 14 January 2016; revised 20 June 2016; accepted 14 September 2016

\begin{abstract}
A numerical investigation is carried out to demonstrate a proof of concept, magnetohydrodynamicsbased active flow control, for mitigation of laminar flow separation over a flat plate due to shock wave-boundary layer interaction. The CERANS-MHD code has been used to solve the governing resistive magnetohydrodynamic equations discretized in finite-volume framework. The AUSM-PW+ flux function is used in modelling the advection terms and central differencing is used in modelling the resistive terms. Powell's source term method is used for divergence cleaning of the magnetic field. The Hartmann number is varied from 0 to 12,000 to effectuate mitigation of flow separation, with the magnetic field applied at the wall and oriented transverse to the flat plate flow direction. Due to the Hartmann effect, flow separation is observed to be suppressed with increase in Hartmann number beyond 6000. However, the overall magnitude of skin friction distribution increases drastically, resulting in large increase in skin friction drag as compared with the non-magnetic case, and is a cause of concern.
\end{abstract}

Keywords. Shock-induced separation; flow control; flat plate; magnetohydrodynamics; CERANS-MHD.

\section{Introduction}

Impingement of an oblique shock wave over a supersonic laminar flow boundary layer on a flat plate can result in local flow separation due to build-up of adverse pressure gradient (APG). This shock-induced flow separation (SIFS) problem is more generally referred to as the shock-boundary layer interaction (SBLI) problem. This problem is very common, for example, on transonic flow over airplane wings and at high altitude operation of a flight vehicle, such as ramjet or scramjet-based devices, where a forebody oblique shock impingement on the inner walls of the air intake can induce a local laminar flow separation, which can lead to mass flow deficiency, engine un-starting and/or loss of thrust.

One of the most commonly used and a traditional method of mitigation of flow separation is applying suction or blowing of flow in the boundary layer. Suction reduces the boundary layer thickness as well as the APG; hence it modifies the local flow structure, thereby energizing the flow, which mitigates separation. However, boundary layer suction has its restrictions, such as requirement of a feedback to the suction mechanism and its related sub-systems where it can be activated only in case of a flow separation

*For correspondence and is highly complex to implement. Also its applicability and effectiveness for wide range of flight operating conditions are limited.

In the present study, a proof of concept, active flow control technique based on magnetohydrodynamic (MHD) flow control has been considered, for studying its applicability and for mitigation of the SIFS over a flat plate. The Hartmann number, defined as the ratio of electromagnetic forces to viscous forces, is the governing non-dimensional parameter considered for the magnetic field variation study performed in this work.

The Hartmann number is defined as follows:

$$
\mathrm{Ha}=B_{\infty} L \sqrt{\frac{\sigma_{e}}{\mu_{\infty}}} .
$$

Here, $B_{\infty}$ is the magnetic field intensity, $L$ is the characteristic length, $\sigma_{e}$ is the freestream electrical conductivity of the flow and $\mu_{\infty}$ is the freestream molecular viscosity.

\section{Active flow control}

Aerodynamic flow control for flight vehicles shall be broadly categorized into mechanical and energetic methods, equivalently called as passive and active techniques, 
depending upon the changes the flow undergoes and the beneficial effects it creates to the vehicle flow field. In case of passive flow control techniques such as vortex generators and boundary layer tripping protrusions, these devices do not require any auxiliary power supply and are easier to maintain and use. However, they can cause high drag and can sometimes cause high flow distortion. In case of semiactive flow control, it is sometimes necessary to actuate the passive protruding device and requires blowing or sucking the boundary layer, however without getting the feedback as to whether the actuation resulted in beneficial effect or worsened the situation.

The energetic means of aerodynamic control or simply called the active flow control techniques are gaining significant attention due to being non-intrusive to flow and flexibility without any moving parts, ease of arrangement/ piloting of the flow control device set-up, high actuation frequency with pulsing mode of operation and effectiveness for wide range of flight conditions. Here an actuating device or actuator is used for energy addition to the flow and hence requires an auxiliary power supply. In this case, local active perturbations created by expending energy on the flow by the actuator bring about global flow field changes with a resultant overall improvement in performance [1]. The main advantage of active flow control is that the actuator can be operated only when necessary as dictated by the flight conditions and can be turned off otherwise. Since the flow control is achieved by an actuating unit, the mechanism of operation is complex, unlike the passive flow control devices, and it is tightly coupled with the physics of the fluid flow.

The energy sources for the energetic methods are direct current arc discharge mechanism, microwave, electrical beam, electric discharge laser beam and MHD systems. In general, the benefits of efficient flow control for an aircraft are high lift, low drag, attached boundary layers, low noise, lower airframe heat-transfer rates, high drag for reentry aerobraking and so on. The payoffs due to such efficient flow control systems directly translate into lower operating cost in case of civil air transports and highly agile manoeuvring aircraft and missiles for military air superiority.

\section{The resistive magnetohydrodynamics governing equations}

The Maxwell equation for magnetic induction coupled with the Navier-Stokes equations describes the resistive magnetohydrodynamics. The resistive MHD governing conservation equations in the Cartesian frame is given by

$$
\frac{\partial \boldsymbol{U}}{\partial t}+\frac{\partial \boldsymbol{F}_{x}}{\partial x}+\frac{\partial \boldsymbol{F}_{y}}{\partial y}+\frac{\partial \boldsymbol{F}_{z}}{\partial z}=\boldsymbol{S}
$$

and the magnetic field is subjected to the solenoid constraint defined by the Gauss law of magnetism given by

$$
\nabla \cdot \boldsymbol{B}=0
$$

where $\boldsymbol{B}$ is the magnetic field vector with Cartesian components $\left(B_{x}, B_{y}, B_{z}\right)$.

The integral forms of Eq. (1) is given by

$$
\frac{\partial}{\partial t} \int_{V} \boldsymbol{U} d V+\int_{A(V)} \nabla . \boldsymbol{F} d A=\boldsymbol{S} . V .
$$

The terms $A$ and $V$ denote, respectively, the area of a cell interface and the volume of the elemental cell for which the fluxes are evaluated. The conserved variable, inviscid, viscous fluxes and source terms in the compact vector form are defined as

$$
\begin{aligned}
& \vec{U}=\left[\begin{array}{c}
\rho \\
\rho \vec{u} \\
\vec{B} \\
\rho e_{t}
\end{array}\right] ; \quad \vec{F}^{(i)} . \hat{n}=\left[\begin{array}{c}
\rho U_{n} \\
\rho U_{n} \vec{u}+p_{t} \cdot \vec{n}-B_{n} \vec{B} \\
U_{n} \vec{B}-\vec{u} B_{n} \\
\left(\rho e_{t}+p\right) U_{n}-B_{n}(\vec{B} . \vec{u})
\end{array}\right] \\
& \overrightarrow{\boldsymbol{F}}^{(v)} \hat{\boldsymbol{n}}=\left[\begin{array}{c}
\mathbf{0} \\
\overline{\bar{\tau}}_{i j} \cdot \hat{n} \\
\overline{\boldsymbol{\beta}}_{i j} \cdot \hat{\boldsymbol{n}} \\
\left(\overrightarrow{\boldsymbol{u}}^{T} \overline{\bar{\tau}}^{\overline{\boldsymbol{n}}} \cdot \hat{\boldsymbol{n}}_{j}-\overrightarrow{\boldsymbol{q}} \cdot \hat{\boldsymbol{n}}\right)
\end{array}\right] ; \quad \boldsymbol{S}=\left[\begin{array}{c}
\mathbf{0} \\
\overrightarrow{\boldsymbol{B}} \\
\overrightarrow{\boldsymbol{u}} \\
\overrightarrow{\boldsymbol{u}} \cdot \overrightarrow{\boldsymbol{B}}
\end{array}\right] \nabla . \boldsymbol{B}
\end{aligned}
$$

where $\boldsymbol{\beta}_{i j}$ is the magnetic shear stress term given by

$$
\boldsymbol{\beta}_{i j}=\boldsymbol{\mu}_{\boldsymbol{B}}\left(\frac{\partial \boldsymbol{B}_{i}}{\partial \boldsymbol{x}_{j}}-\frac{\partial \boldsymbol{B}_{j}}{\partial \boldsymbol{x}_{i}}\right) \quad \text { and } \quad \boldsymbol{\mu}_{\boldsymbol{B}}=\frac{1}{\mu_{o} \sigma_{\boldsymbol{e}}}
$$

Here $\rho$ is the density of the fluid and $\boldsymbol{u}$ is the velocity field vector with Cartesian components $(u, v, w)$. The terms $U_{n}$ and $B_{n}$ are the contra-variant velocity and magnetic fields, respectively, defined as $\boldsymbol{U}_{\boldsymbol{n}}=\boldsymbol{u} . \boldsymbol{n}=u \hat{\boldsymbol{n}}_{\boldsymbol{x}}+v \hat{\boldsymbol{n}}_{\boldsymbol{y}}+w \hat{\boldsymbol{n}}_{z}$ and $\boldsymbol{B}_{\boldsymbol{n}}=\boldsymbol{B} . \boldsymbol{n}=\boldsymbol{B}_{x} \hat{\boldsymbol{n}}_{\boldsymbol{x}}+\boldsymbol{B}_{\boldsymbol{y}} \hat{\boldsymbol{n}}_{\boldsymbol{y}}+\boldsymbol{B}_{z} \boldsymbol{n}_{z}$

The mechanical pressure $p$, also called as the fluid static pressure, which is the thermodynamic or mechanical pressure term, and the magnetic pressure term $\frac{\boldsymbol{B} \cdot \boldsymbol{B}}{2}$ are grouped together and their sum is denoted by a total pressure term as $p_{t}$ :

$$
p_{t}=p+\frac{\boldsymbol{B} \cdot \boldsymbol{B}}{2}=p+\frac{1}{2}\left(B_{x}^{2}+B_{y}^{2}+B_{z}^{2}\right)
$$

The total energy is represented as

$$
\begin{aligned}
& \rho e=\frac{1}{2} \rho u^{2}+\frac{1}{2} B^{2}+\frac{p}{\gamma-1} \\
& \rho e=\frac{1}{2} \rho\left(u^{2}+v^{2}+w^{2}\right)+\frac{1}{2}\left(B_{x}^{2}+B_{y}^{2}+B_{z}^{2}\right)+\frac{p}{\gamma-1}
\end{aligned}
$$

where $e$ is the total energy per unit mass of the fluid. In the above equations, the actual magnetic field is defined to be equal to $B=B / \mu_{o}$, where $\mu_{o}$ is the magnetic permeability of free space, equal to $4 \pi \times 10^{-7} \mathrm{~N} / \mathrm{A}^{2}$, and $\sigma_{e}$ is the electrical conductivity of the fluid. 
Here the fluid is considered as a perfectly conducting plasma, i.e., the flow is fully ionized and hence the electrical conductivity of the gas is practically infinite. However, for numerical purposes, the value of $\sigma_{e}$ is taken as $1.6 \times 10^{10} \mathrm{mho} / \mathrm{m}$ [2]. The governing equations implemented in the work essentially consider that the external flow medium is fully ionized such that the influence of applied magnetic field on the flow field and vice versa is undiminished. This condition is a prerequisite for the currently implemented model and within this purview, the assumption shall be considered reasonable.

\subsection{Divergence-free magnetic field constraint, $\nabla \cdot B=0$}

In case of solution with discontinuities, the initial divergence-free solution can accumulate numerical truncation errors, leading to unphysical evolution, thereby resulting in large values of divergence of the magnetic field. Hence to satisfy the divergence-free magnetic field condition all through the evolution of numerical solution, a source term vector $S$ is required to be added in Eq. (1). It shall be noted that the requirement of divergence-free constraint is inherently present in the divergence form of ideal MHD equations. However, due to possibilities of coincidence of eigenvalues and existence of multiple waves across a discontinuity, numerically, an initial divergence-free magnetic field need not maintain divergence-free condition during time evolution, though the governing equations guarantee this at all times. In case of smooth solutions, this condition is met and the divergence errors do not grow, if the numerical truncation errors are small.

The source term $\boldsymbol{S}$ can be evaluated either as volume source term or as a projection of flux. In the eight-wave source term method developed by Powell [3], $\boldsymbol{S}$ is a nonconservative source term proportional to $\nabla . \boldsymbol{B}$, which is used to remove the singularity of Jacobian matrix of the ideal MHD system.

\subsection{Wave speeds of ideal MHD system}

The ideal MHD system, which defines the convective part of the resistive MHD equation, is hyperbolic in nature; however it is not strictly hyperbolic due to non-distinct eigenvalues. Hence some of the wave speeds may be equal and the eigenvalues can coincide. This characteristic nature of ideal MHD equations leads to solutions of Riemann problems involving both a shock wave and a rarefaction wave existing across a discontinuity, also called as the compound wave. The eigenvalues of the flux-Jacobian of ideal MHD system yields eight wave speeds and are given by

$$
\begin{aligned}
& \lambda_{e}=\boldsymbol{U}_{\boldsymbol{n}} ; \lambda_{d}=\boldsymbol{U}_{\boldsymbol{n}} ; \quad \lambda_{s}=\boldsymbol{U}_{\boldsymbol{n}} \pm c_{s} ; \quad \lambda_{A}=\boldsymbol{U}_{\boldsymbol{n}} \pm c_{A} \quad \text { and } \\
& \quad \lambda_{f}=\boldsymbol{U}_{\boldsymbol{n}} \pm c_{f} .
\end{aligned}
$$

Here, $\lambda_{e}$ is the eigenvalue for entropy wave, $\lambda_{d}$ is the eigenvalue for magnetic-flux divergence wave, $\lambda_{s}$ is the eigenvalue for the slow magneto-acoustic wave, $\lambda_{A}$ is the eigenvalue for the Alfven wave and $\lambda_{f}$ is the eigenvalue for fast magneto-acoustic wave and these waves satisfy the condition $c_{s} \leq c_{A} \leq c_{f}$. Alfven waves are purely transverse waves, which are formed due to resistance of transverse motion by the magnetic field, and they propagate at the Alfven velocity, $c_{A}=\boldsymbol{B} / \sqrt{\rho}$. It has to be noted that the fast/slow magnetosonic waves are genuinely non-linear waves and the Alfven/entropy waves are linearly degenerate. The fast and slow waves are given by

$c_{f, s}=\sqrt{\frac{1}{2}\left\{\frac{\gamma p+|\boldsymbol{B}|^{2}}{\rho} \pm \sqrt{\left(\frac{\gamma p+|\boldsymbol{B}|^{2}}{\rho}\right)^{2}-\frac{4 \gamma p \boldsymbol{B}}{\rho^{2}}}\right\} / 2 \rho .}$

If the magnetic flux term is zero then the eigenvalues reduce to the perfect gas eigenvalues. The eigenvalue corresponding to the fast magneto-acoustic wave speed is used for obtaining the time step required for temporal evolution.

\subsection{Numerical procedure adopted in CERANS- MHD}

The ideal MHD governing equations, which define the coupled fluid flow equations, i.e., the Navier-Stokes equations and the Maxwell equations of electromagnetics, have been implemented in the finite-volume solver CERANS (compressible Euler/Reynolds-averaged NavierStokes) [4] and called as CERANS-MHD [5]. It is an edgebased solver where a grid format-independent geometric information is provided to the solver using a grid preprocessor.

The AUSM-PW+ flux function $[6,7]$ is used for modelling the advection terms. The resistive terms were discretized based on central differencing approximation. The Powell source term method [3] is used for divergence cleaning of the magnetic field. The flow gradients were evaluated using the method of weighted least squares. Second order spatial accuracy is obtained using the procedure of reconstruction and the Barth min-max limiter [8] is used to preserve monotonicity. The code has been validated for standard complex test cases available in literature with very good comparative performance.

\section{Magnetohydrodynamic shock boundary layer interaction problem}

A schematic of the isolated problem for SIFS control using MHD is shown in figure 1 . It shows an imaginary wedge that generates an oblique shock, which enters the actual 


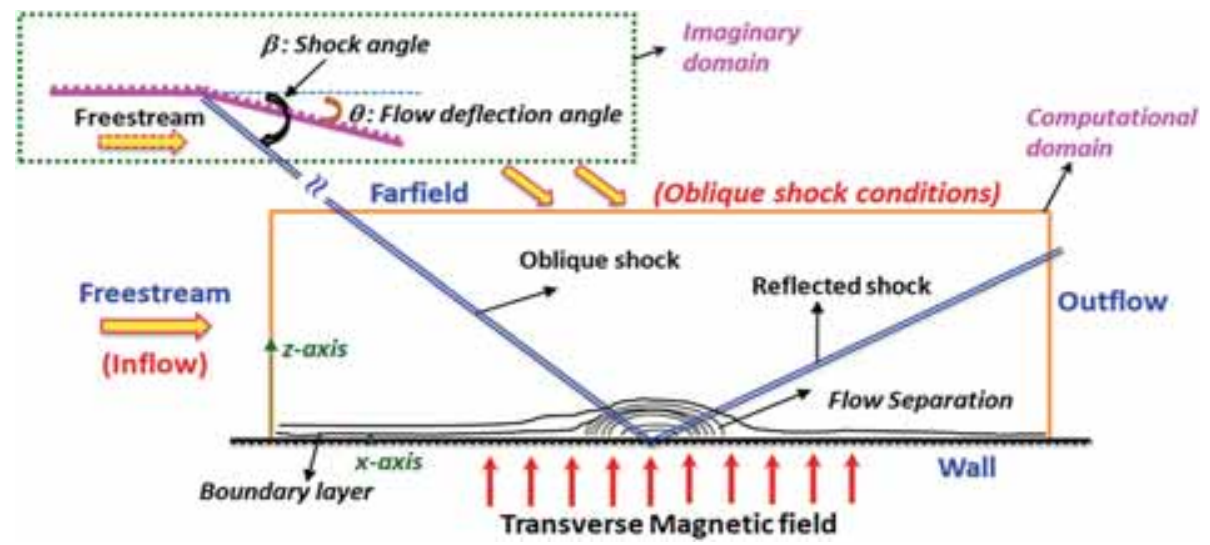

Figure 1. Schematic of the MHD shock-boundary layer interaction problem.

computational domain through the far-field boundary and interacts with the wall boundary layer, thereby inducing flow separation. The direction of transverse magnetic field is as depicted in figure 1 and acts along the positive $z$ direction (vertical).

In order to demonstrate flow separation control using $\mathrm{MHD}$, an exclusive qualitative comparative study had been carried out based on the work of Ekaterinaris [9, 10], where flow separation control of a laminar boundary layer due to interaction of an oblique shock impingement over a flat plate under the influence of magnetic field had been analysed. The numerical method used in Ekaterinaris [9, 10] is a fifth order conservative finite-difference WENO scheme with a Roetype approximate Riemann solver for computing the inviscid fluxes and Powell's source term method [3] for preserving the divergence-free magnetic field constraint.

In this work, qualitative corroboration of the results obtained using CERANS-MHD code with information available from Ekaterinaris $[9,10]$ is appraised. Moreover, this test case is considered for numerical investigation, only as a representative problem for demonstrating the proof of concept MHD flow control.

\section{Computational grid}

Three structured grids were generated for addressing this problem and shall be hereafter referred to as coarse, medium and fine grids. The size of the coarse grid is $171 \times 4 \times 101$ (171 points along the length of the plate, 4 points along the span-wise direction, which is directed into the plane of the paper, and 101 points away from the wall) and amounts to about 69,000 points. The sizes of medium and fine grids are $171 \times 4 \times 151$ and $171 \times 4 \times 201$, respectively, amounting to 103,000 and 138,000 points.

The computational mesh for the fine grid is shown in figure 2 . In all the grids, the far-field boundary is so chosen that the leading edge shock from flat plate is accommodated completely within the computational domain and hence the

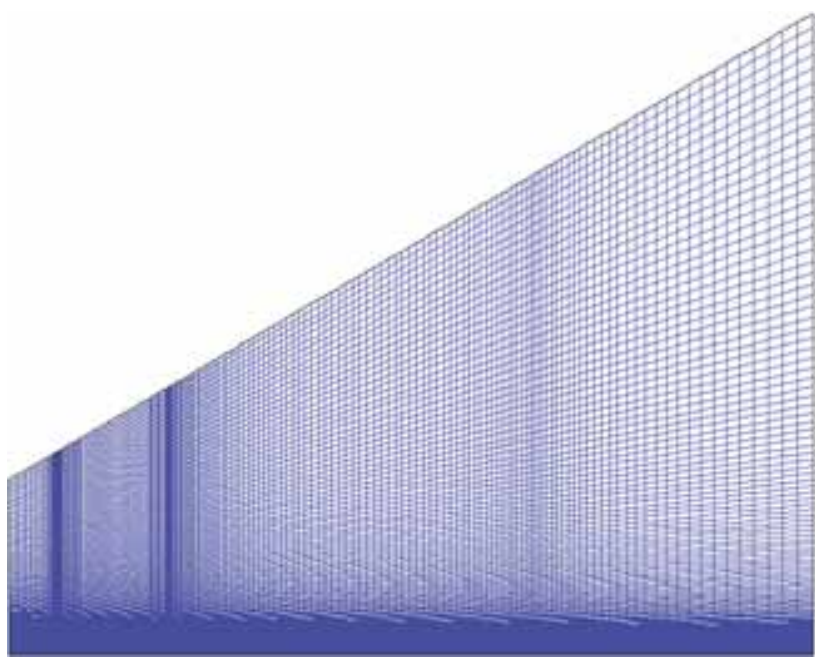

Figure 2. Grid for MHD SBLI problem.

height of the far field at outflow is greater than that at inflow. Necessary clustering near the wall, at the flat plate leading edge, around the oblique shock location at far field and around the shock impingement location has been provided.

As a transverse magnetic field is applied to an electrically conducting fluid flow over a wall surface, a specific boundary layer known as the Hartmann boundary layer is formed near the wall region, where the Lorentz forces and the viscous forces balance each other. In case of MHD flows, the entire shear stress is concentrated within the Hartmann layer and the thickness of this layer is roughly inversely proportional to $\mathrm{Ha}$. In case of laminar MHD flows, the requirement of near-wall spacing is dictated by the minimum of the Hartmann boundary layer thickness and the velocity boundary layer thickness. The velocity boundary layer thickness is inversely proportional to $\operatorname{Re}_{x}^{1 / 2}$. Since the flow Reynolds number and the highest Hartmann number for the present study are of the same order $\sim 10,000$, the Hartmann layer thickness is expected to be much smaller than the boundary layer thickness. Hence the 
near-wall spacing requirement for this case, for the medium and fine grids, is decided based on the highest Hartmann number of 12,000 .

\section{Flow conditions and simulation details}

The flow conditions considered in this study are Mach number 3 and oblique shock angle of $30^{\circ}$ (corresponding to a wedge angle $\theta_{\text {wedge }}=12.775^{\circ}$ ). The flow Reynolds number is $10^{4}$ per unit length. The post-oblique shock conditions applied at the far-field boundary are pressure ratio of 2.459 , density ratio of 1.862 and temperature ratio of 1.320. For a freestream temperature of $300 \mathrm{~K}$, the density of the flow is $1.72 \times 10^{-4} \mathrm{~kg} / \mathrm{m}^{3}$ and the freestream pressure is $14.8 \mathrm{~N} / \mathrm{m}^{2}$. The post-shock Mach number is 2.367 and the total velocity $(q)$ can be obtained based on the acoustic speed and the temperature ratio. Hence the postoblique-shock velocity components are

$$
\begin{gathered}
u=q \cos \left(\theta_{\text {wedge }}\right) \\
v=0 \\
w=-q \sin \left(\theta_{\text {wedge }}\right)
\end{gathered}
$$

These post-oblique-shock conditions are applied at the farfield boundary, freestream conditions are applied at the inflow and simple extrapolation is used at the outflow.

The Hartmann number is varied from 0 to 12,000 (based on length scale of $1 \mathrm{~m}$ ) in this study. Corresponding uniform magnetic field intensity (in Tesla) of 0 to $0.12 \times 10^{-3}$ $\mathrm{T}$ is applied at the wall directed into the flow field, transverse to the flow direction. Further, at the wall, adiabatic, no slip boundary condition and zero normal pressure gradient conditions are imposed. The flow is assumed fully ionized, with an electrical conductivity of $1.6 \times 10^{10} \mathrm{mho} /$ $\mathrm{m}$. The molecular viscosity is obtained using Sutherland's formula and the freestream molecular viscosity is $1.7895 \times 10^{-5} \mathrm{~kg} /(\mathrm{ms})$. In general, typical values of Hartmann number for aerodynamic flows range from a few tens to thousands, depending upon the magnetic configuration being used and the practically achievable values of fluid electrical conductivity.

\section{Results and discussions}

\subsection{Grid convergence study}

Among the three grids used for numerical study, the first wall grid spacing of $0.25 \mathrm{~mm}$ for the coarse grid is decided roughly based on the velocity boundary layer thickness. A numerical study was performed for laminar supersonic flow past flat plate for $\mathrm{Ha}=0, \mathrm{Re}=10,000$ without the incident oblique shock. The details of the results are presented in the next section. The near-wall grid resolution was observed to be adequate for resolving the boundary layer and there were about 40 points within the boundary layer thickness at a length of about 1.5-2 $\mathrm{m}$ (where the oblique shock is expected to impinge on the wall) from the flat plate leading edge.

In case of the medium and fine grids, the first wall grid spacing was decided based on the highest Hartmann layer thickness of 12,000 and considered equal to 50 and $25 \mu \mathrm{m}$, respectively. For the non-magnetic laminar supersonic flat plate flow, the number of points within the boundary layer at $2 \mathrm{~m}$ length is 100 in the medium grid and 130 in the fine grid. It was observed that the results of coarse grid were having marginal difference with that of the medium and fine grid. However there were no significant differences between the results due to medium and fine-grid solutions. Hence the results are considered grid independent for these grids and the results and discussion are presented only for fine-grid solution.

7.1a Case (i): flow past a flat plate, non-magnetic case Computations were performed initially without magnetic field $(\mathrm{Ha}=0)$ and without oblique shock impingement. This case corresponds to the simple supersonic laminar flow past a flat plate with a developing boundary layer for which the simulated skin friction can be compared to analytical expression using the Blasius solution given by

$$
c_{f}=\frac{0.664}{\sqrt{R_{x}}}
$$

where $R e_{x}$ is the local Reynolds number based on the length $x$ from the leading edge of the flat plate.

The plot of local skin friction coefficient obtained using CERANS-MHD without shock boundary interaction along the flat plate length is shown in figure 3 . This case is also called as the laminar supersonic flat plate boundary layer flow represented here as FPBL. The Blasius skin friction coefficient is plotted for comparison. It can be observed that the skin friction variation due to present simulation reproduced the exact results due to Blasius skin friction coefficient distribution and hence establishes the accuracy of the numerical method.

7.1b Case (ii): oblique shock boundary layer interaction and without magnetic field This case corresponds to the laminar oblique SBLI over the developing flat plate boundary layer in the absence of magnetic field. The simulation result for this case is depicted in figure 4 , in which the density contours are plotted, with flow streamlines depicting large SIFS bubble. From the flow field it can be observed that there are two interacting shocks, one from the flat plate leading edge and the other due to the imposed oblique shock condition. The oblique shock due to the imposed condition impinges on the wall and gets reflected, causing a local pressure rise, resulting in an APG sufficient enough to cause flow reversal and subsequently, a large flow separation. The flat plate leading edge shock and the 


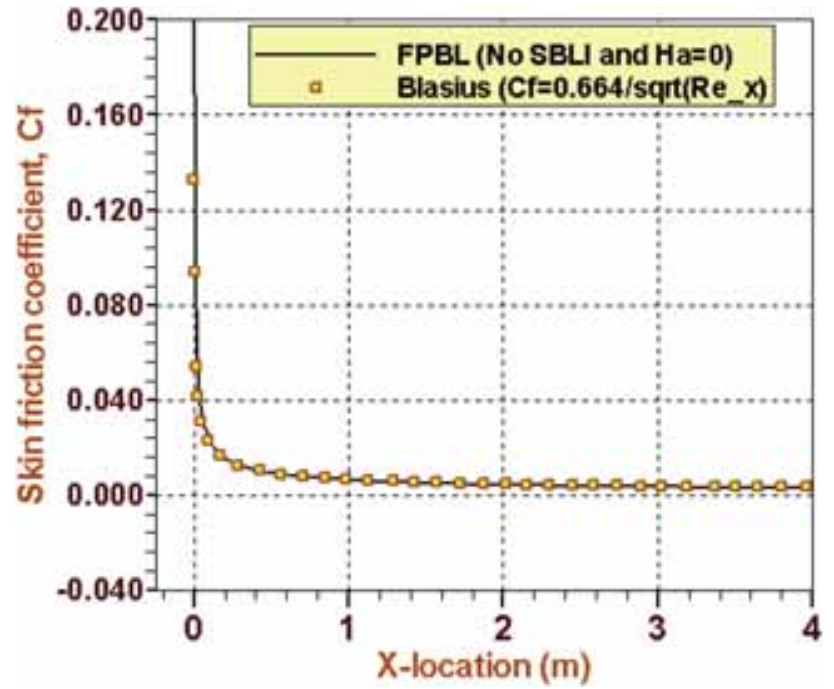

Figure 3. Comparison of skin friction coefficients.

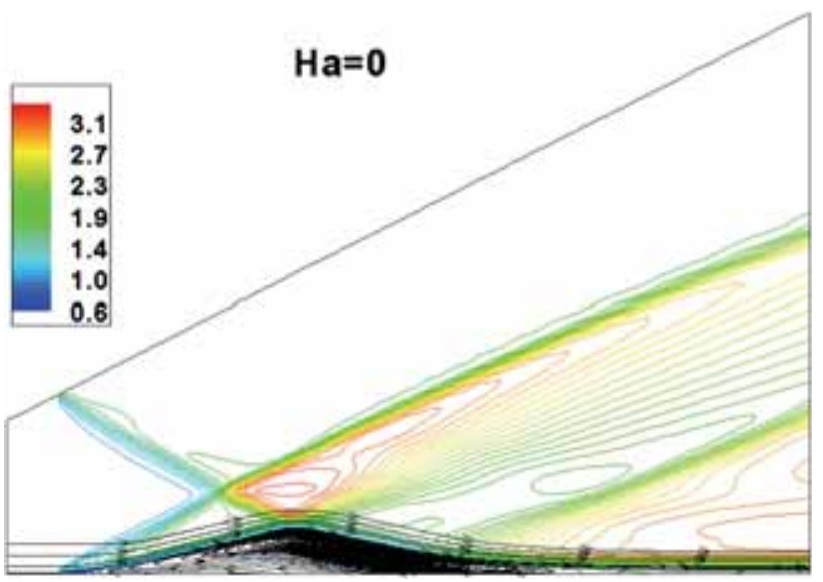

Figure 4. Density contours and shock-induced flow separation.

reflected oblique shock can be observed to exit the computational domain through the outflow boundary. It was observed that the flow separation zone is mildly unsteady.

Due to flow separation, the boundary layer lifts up and the dividing streamline traces a hump-like convex structure, over which the flow further expands downstream till the boundary layer reattaches. The separation bubble at the upstream region of the separation zone is slanted upwards and the downstream end of the bubble is at an elevated position. The flow structure and the separation zones appear highly complex, due to formation of multiple separation bubbles. The hump structure of the separation bubble in the interaction zone has a concave turning, which causes the flow expansion over the dividing streamline to terminate with a reflected oblique shock. Further downstream along the dividing streamline, up to the reattachment junction, a

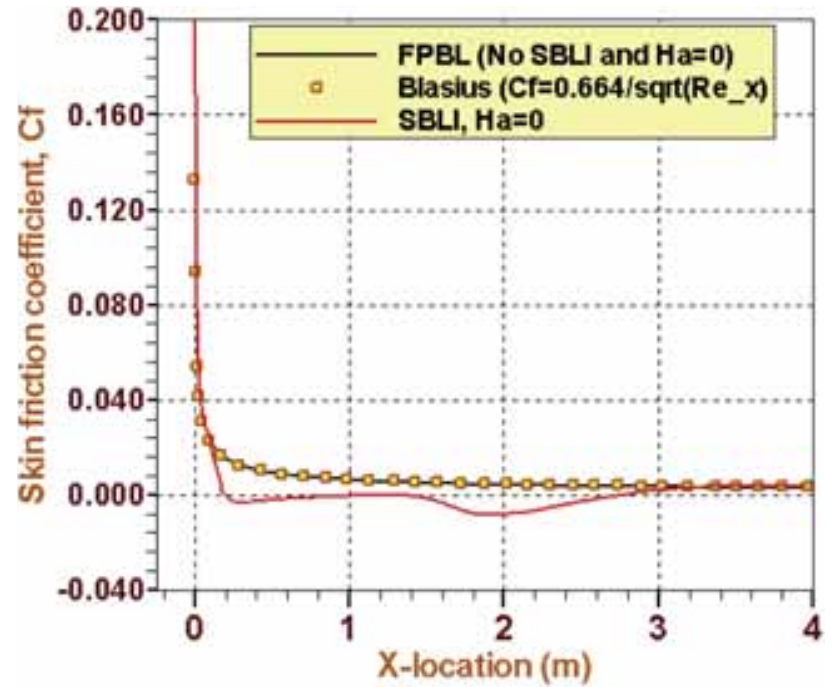

Figure 5. Comparison of various non-magnetic skin friction coefficient distributions.

mild expansion fan can be observed; thereafter, due to concave turn at the reattachment junction, a strong reflected oblique shock emerges and exits through the outflow boundary. It can be observed that the separation bubble had almost reached the flat plate leading edge, where the leading edge shock originated.

The plot of skin friction coefficient $\left(c_{f}\right)$ depicting the flow separation is shown in figure 5. From the plot, it can be observed that the upstream incipient separation location is about $0.194 \mathrm{~m}$ and the separation region continues till 2.754 $\mathrm{m}$. A local zone of positive skin friction has been observed from 1.11 to $1.22 \mathrm{~m}$.

7.1c Case (iii): oblique shock boundary layer interaction with magnetic field interaction This case corresponds to applied transverse magnetic field for Hartmann numbers of $3000,6000,9000,10,000$ and 12,000 . The flow is considered fully ionized and the wall is considered perfectly insulated. Due to large gradient of electrical conductivity near the insulated wall, an electric field is generated, which causes electric current to circulate inside the boundary layer. Due to a positive current density in the boundary layer, the flow in the boundary layer is accelerated by the Lorentz force, which flattens the velocity profiles, resulting in flow attachment and mitigation of flow separation.

\subsection{Streamlines and density contours}

The density contours and the flow streamlines for these cases have been presented in figures 6-10. Due to application of magnetic field, it can be observed that as the Hartmann number is increased, the flow separation bubble size reduces and the flow tends to get fully attached to the wall. For Ha beyond 9000, a complete flow attachment at the SBLI zone has been observed. This result is 


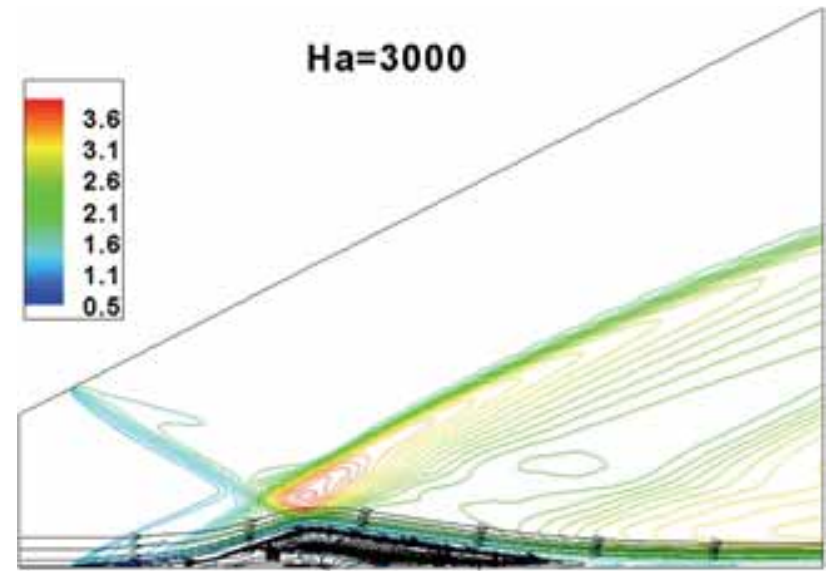

Figure 6. Density contours and streamlines for $\mathrm{Ha}=3000$.

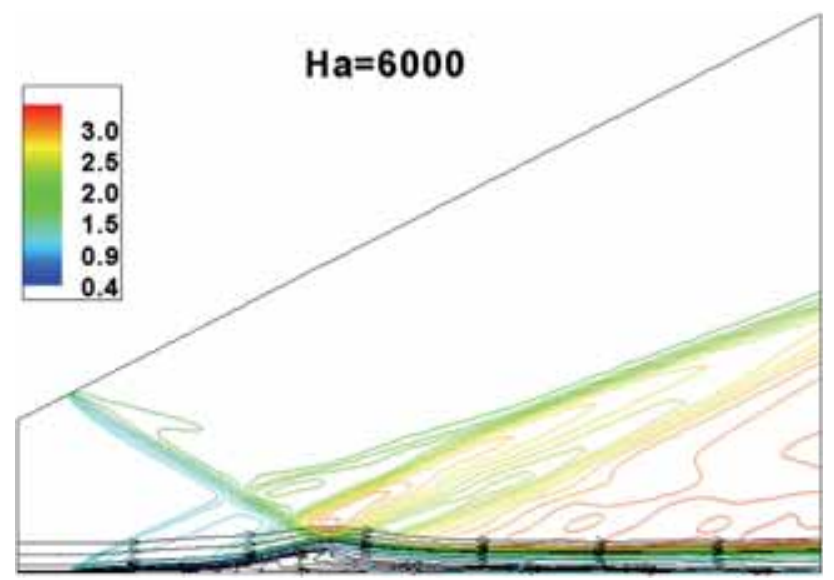

Figure 7. Density contours and streamlines for $\mathrm{Ha}=6000$.

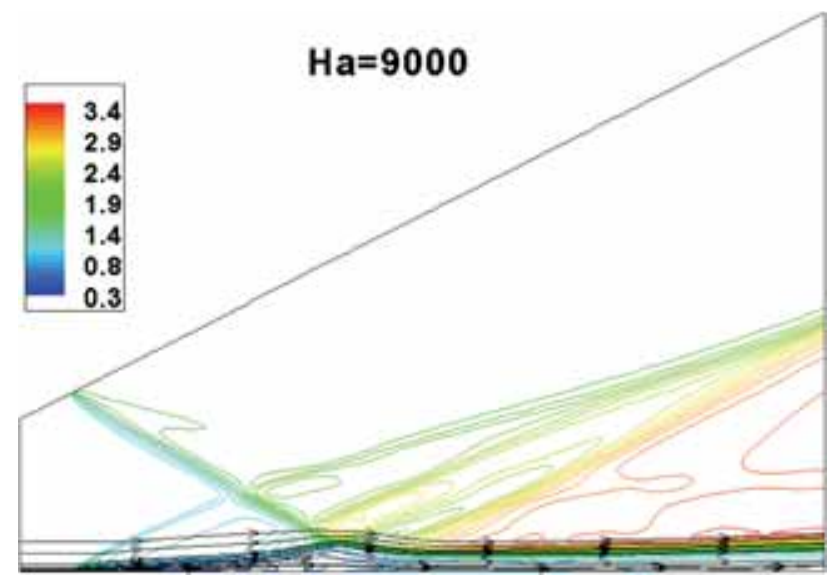

Figure 8. Density contours and streamlines for $\mathrm{Ha}=9000$.

qualitatively similar to the results of Ekaterinaris [9], where complete flow attachment has been discussed for $\mathrm{Ha}=10,000$. The reflected shock and expansions depict a

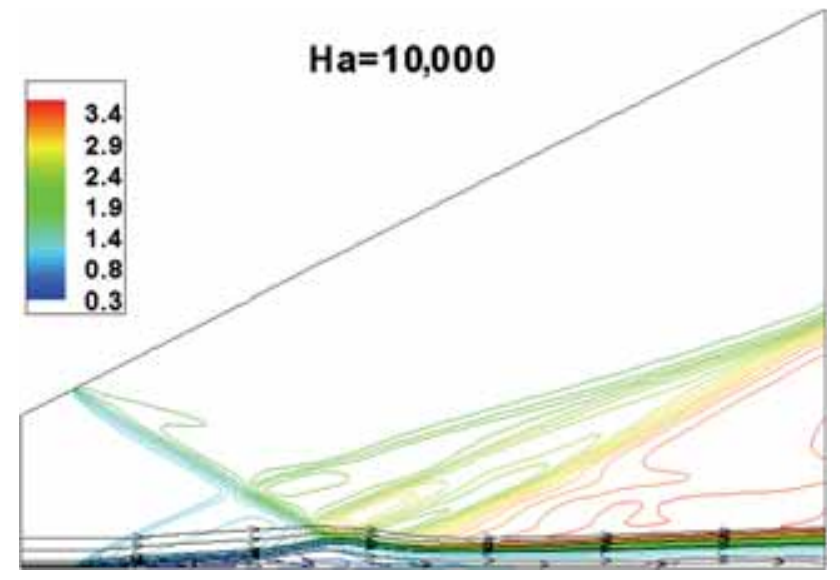

Figure 9. Density contours and streamlines for $\mathrm{Ha}=10,000$.

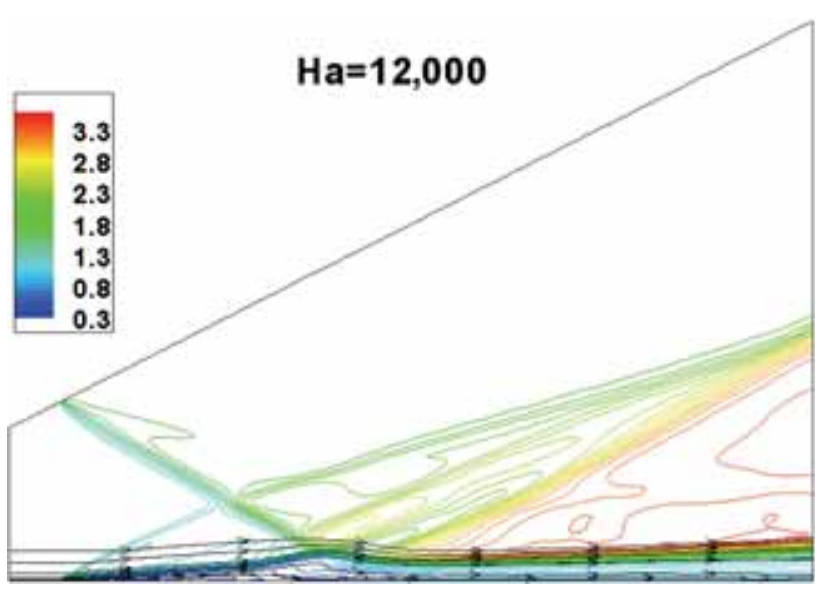

Figure 10. Density contours and streamlines for $\mathrm{Ha}=12,000$.

very complex pattern for $\mathrm{Ha}$ of 0 and 3000 . However, for $\mathrm{Ha}=6000$ and beyond, the reflected shock tends to merge in the downstream region.

A qualitative comparison with Ekaterinaris [9, 10] revealed that the mitigation of flow separation occurs at similar Hartmann numbers (9000 for CERANS-MHD and 10,000 for Ekaterinaris [9]) in spite of the noticeable differences in the prediction of vortical structures, mainly attributed to the geometrical differences between the configuration used and the spatial order of accuracy, which is 5 th order in case of reference article and 2 nd order in the present case.

\subsection{Wall pressure distribution}

The wall pressure distributions $\left(p / p_{\infty}\right)$, for the flat plate boundary layer flow and SBLI, with and without imposed transverse magnetic field, are depicted in figure 11. The 


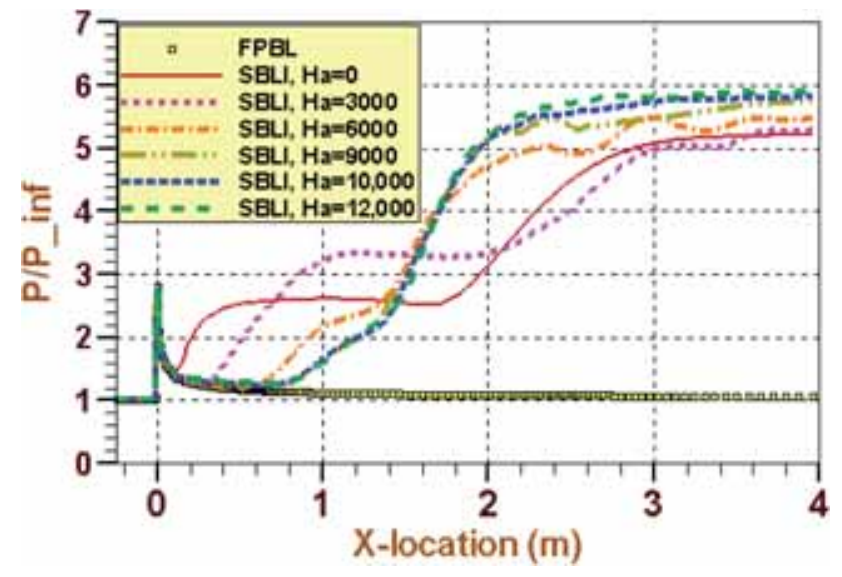

Figure 11. Wall pressure distribution for various Hartmann numbers.

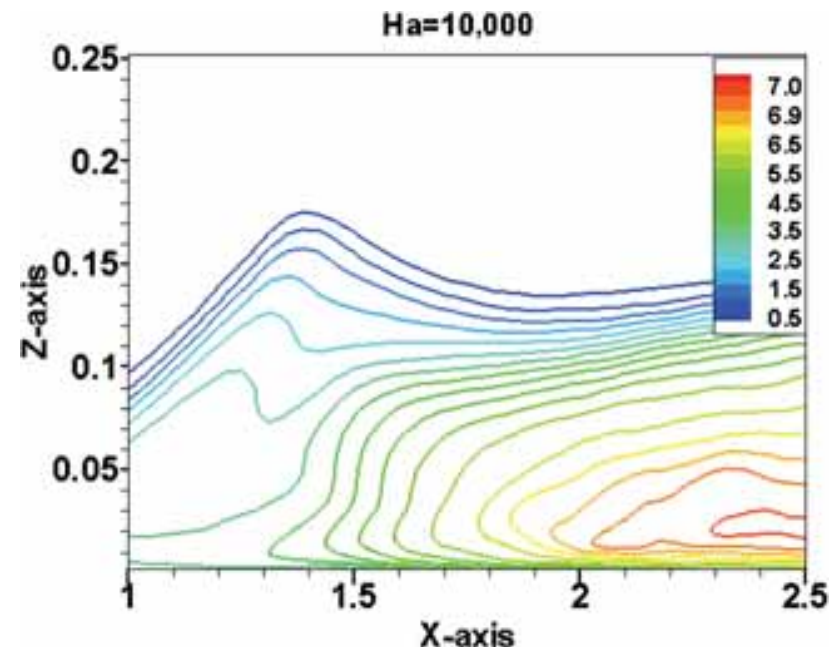

Figure 12. Normalized magnetic field intensity contours for $H a=10,000$.

pressure rise due to the flat plate leading edge shock is about 2.8. For the FPBL case, the wall surface pressure asymptotically reaches the zero-pressure-gradient profile

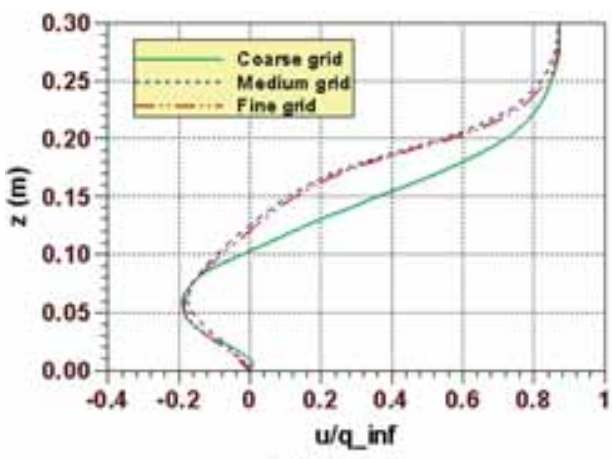

(a) $\mathrm{Ha}=0$ and recovers to freestream pressure. In case of non-magnetic SBLI flow (solid line), the pressure distribution shows a couple of APG regions, one at around $0.2 \mathrm{~m}$ and the other at about $1.7 \mathrm{~m}$. They are, respectively, due to the incipient separation located at $0.194 \mathrm{~m}$ (figure 5) and the oblique shock impingement on the flat plate boundary layer.

In case of SBLI with imposed magnetic field, for $\mathrm{Ha}=3000$, the location of first APG due to flow separation can be observed to move downstream to about $0.35 \mathrm{~m}$; however, the pressure in the plateau region after the pressure jump is about 3.3 and is higher than in the $\mathrm{Ha}=0$ case, which is about 2.6. The second APG occurs at about 2 $\mathrm{m}$ and reaches a plateau downstream to a value of 5.3, similar to the $\mathrm{Ha}=0$ case. With $\mathrm{Ha}=6000$, the location of first APG can be observed to move further downstream to about $0.6 \mathrm{~m}$ and till about this distance, the pressure distribution resembles the FPBL distribution. For this case, the two APGs can be observed to be consecutive, and the second APG due to oblique shock is steeper than the first APG. For the case of $\mathrm{Ha}=9000$ and beyond, the pressure distribution overlaps without any significant changes in the interaction zone. Also the wall pressure downstream of the interaction zone for Ha beyond 6000 is significantly higher than that of the $\mathrm{Ha}=0$ case as can be observed from figure 11. The trend of pressure distribution for the SBLI problem with various imposed magnetic fields is observed to be comparable to that of $\mathrm{Su}$ et al [11] for an analogous problem.

It is interesting to note that at an angle of attack, the modified pressure distribution over the wall due to the imposed magnetic field can bring about significant changes in the overall parameters such as the lift and drag forces as compared with the non-magnetic SBLI problem.

\subsection{Magnetic field contours $(\mathrm{Ha}=10,000)$}

The contours of normalized magnetic field intensity for $\mathrm{Ha}=10,000$ in the interaction region are depicted in figure 12. A high concentration of magnetic field intensity can be observed near the wall, which diffuses gradually away

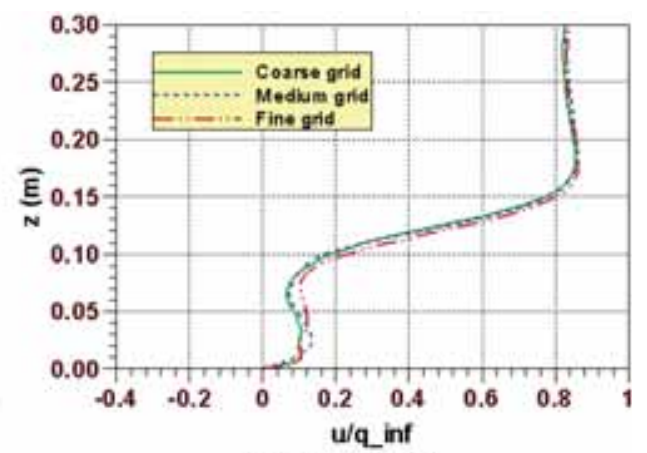

(b) $\mathrm{Ha}=12,000$

Figure 13. Comparison of velocity profiles at $x=1.5 \mathrm{~m}$ for various grids. (a) $\mathrm{Ha}=0$, (b) $\mathrm{Ha}=12,000$ 
from the wall. Downstream of the interaction zone, beyond $1.5 \mathrm{~m}$ length, due to freezing of the magnetic field with the flow field, an increase in magnetic field intensity can be observed. The contours of magnitude of magnetic field are observed to be qualitatively similar to those of Ekaterinaris [10].

\subsection{Boundary layer profiles}

Comparison of velocity profiles for the three grids, for Hartmann number of 0 and 12,000, at the axial location of $x=1.5 \mathrm{~m}$, which is around the middle of the SBLI region, is depicted in figure 13a and b, respectively. At the outset, it can be observed that the velocity profiles look almost similar for the three grids. Flow reversal has been captured by all the grids in case of $\mathrm{Ha}=0$ and the profiles due to medium and fine grids almost overlap. The coarse-grid profile can be observed to show significant deviation beyond $z=0.08 \mathrm{~m}$. In case of $\mathrm{Ha}=12,000$, in the nearwall zone, the velocity profiles for all the three grids appear identical and the flow is attached, having positive velocity due to Hartmann effect. However, near the wall zone, for $z<0.05 \mathrm{~m}$, small difference in velocity profiles can be observed. Considering the close resemblance of the medium and fine-grid profiles, the fine-grid results shall be considered almost grid independent.

The plots of velocity boundary layer profiles in all the above cases at various axial stations are shown in figures $14-17$. The velocity profile due to the laminar supersonic flat plate boundary layer without SBLI for $\mathrm{Ha}=0$ is also plotted with symbols in all these plots. At locations upstream of the interaction zone, this profile can be made use of for comparative purposes and is hereafter referred to as flat-plate boundary layer case or simply FPBL or no SBLI and $\mathrm{Ha}=0$ in the legends of various graphs.

The velocity profiles in figure 14 correspond to $x$ location of $0.205 \mathrm{~m}$, where the incipient flow separation occurs

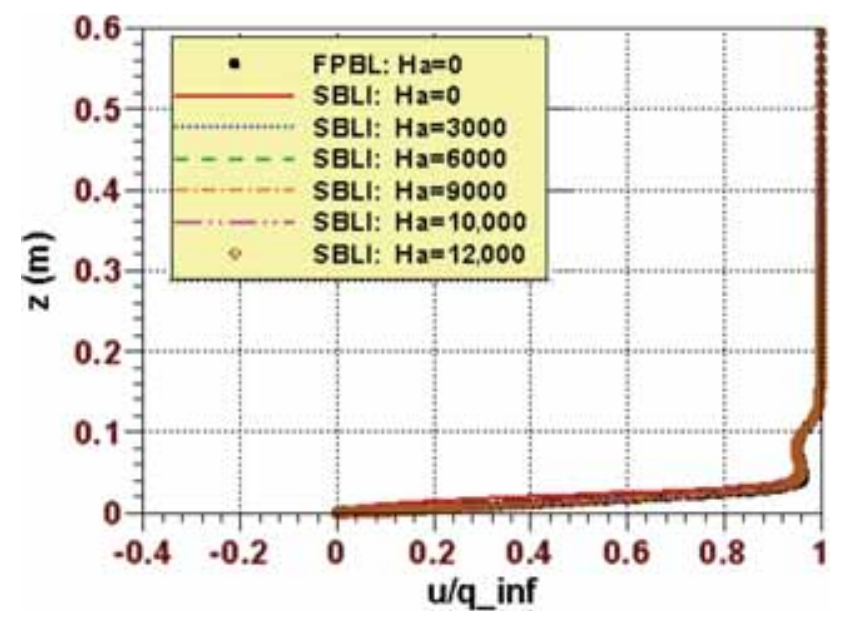

Figure 14. Velocity profiles at $x=0.205 \mathrm{~m}$.

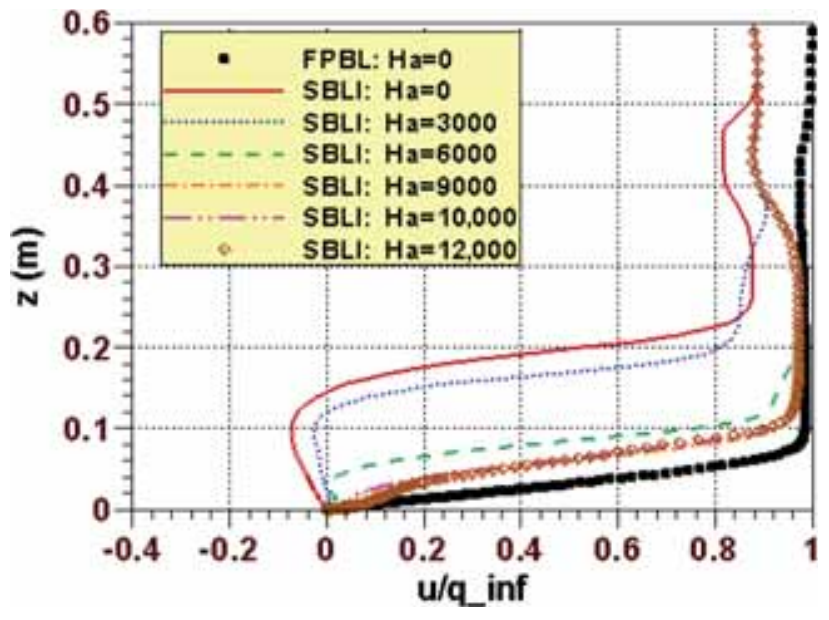

Figure 15. Velocity profiles at $x=1.0 \mathrm{~m}$.

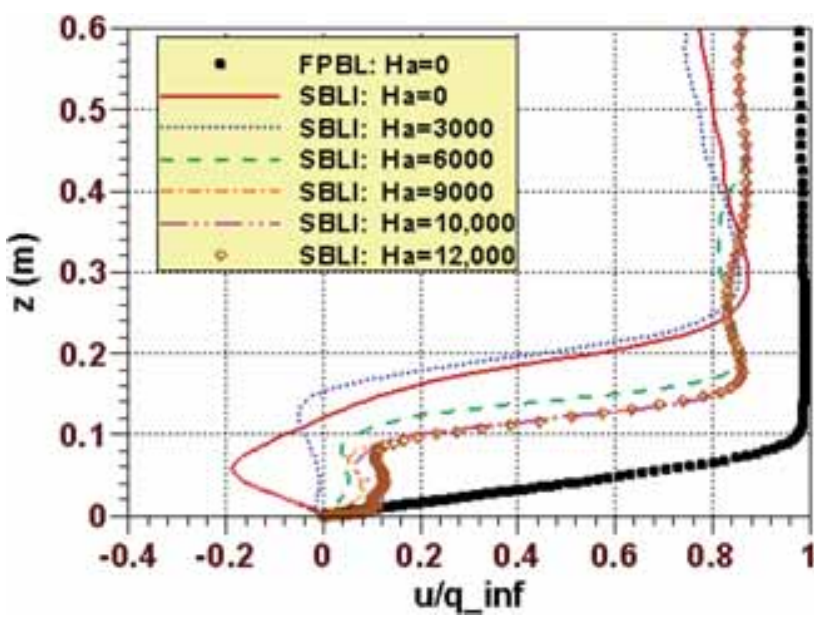

Figure 16. Velocity profiles at $x=1.5 \mathrm{~m}$.

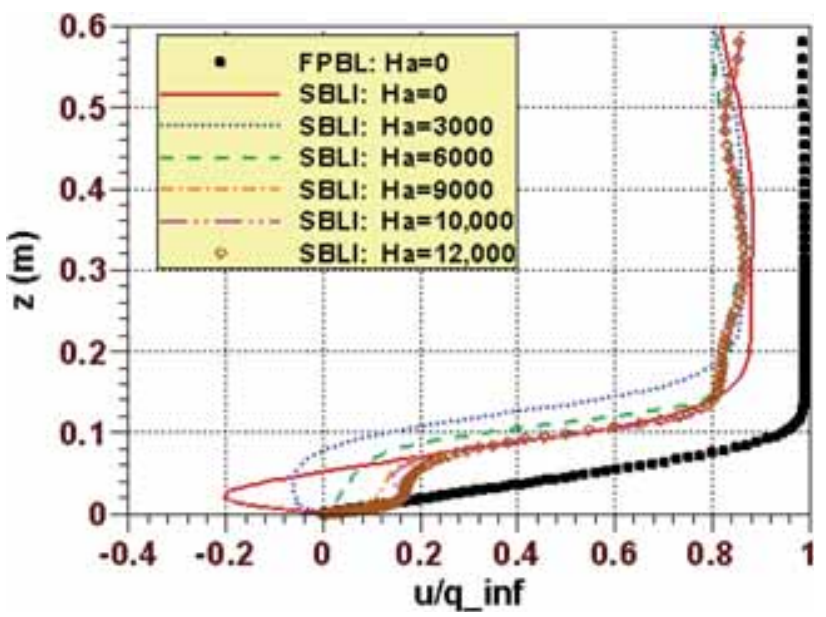

Figure 17. Velocity profiles at $x=2.0 \mathrm{~m}$. 
for the non-magnetic-field case $(\mathrm{Ha}=0)$ and the velocity gradient is almost zero near the wall. At the same station of $x=0.205 \mathrm{~m}$, velocity profiles for all non-zero magnetic field cases have collapsed and resemble the same irrespective of the magnitude of the imposed magnetic field strength, and furthermore are identical to the FPBL profile. Also there is no incipient separation for the non-zero magnetic case at this location. It is interesting to note that due to application of magnetic field, the velocity profile can be observed flattened because of Hartmann-like effect and the flow develops like the FPBL without any separation.

Figure 15 shows the velocity profiles at $x=1.0 \mathrm{~m}$, where the SBLI is strong. The non-magnetic case shows a very large flow separation and the height of dividing streamline is about $z=0.13$. The peak magnitude of flow reversal velocity is about -0.08 times the freestream velocity. By applying magnetic field, for $\mathrm{Ha}=3000$, the flow separation still persists, however with lower magnitude of reverse flow velocity. With further increase of Hartmann number to 6000, the flow reversal disappears. By further increase of Hartmann number to 9000 and beyond, the profile tends towards the FBLI profile.

At a location of $1.5 \mathrm{~m}$ from the leading edge of the plate, all the velocity profiles have been observed to be flatter than that of the $1.0 \mathrm{~m}$ case as shown in figure 16 . However, the flow reversal still persists and the flow reversal velocity is -0.18 for the non-magnetic case and -0.07 for $\mathrm{Ha}=3000$. For $\mathrm{Ha}=6000$ and beyond, the flow reversal disappears; the velocity profile however does not resemble the FPBL profile.

At the axial location of $2.0 \mathrm{~m}$, the velocity profile as depicted in figure 17 shows a complete reattachment for $\mathrm{Ha}=6000$ and above, where the SBLI with $\mathrm{Ha}=0$ case shows a peak flow reversal velocity of -0.2 with inflection occurring at $z / L=0.003$. Here also, for $\mathrm{Ha}=6000$ and beyond, the flow is completely attached.

\subsection{Local skin friction distribution}

From figures $14-17$, it can be clearly observed that between Ha of 6000 and 9000 the flow separation is getting controlled and for $\mathrm{Ha}=9000$ and beyond, the imposed magnetic field completely mitigates the flow separation. A plot of variation of local skin friction along the wall for various magnetic field strengths is shown in figure 18 .

As the magnetic field is increased, the zone of flow separation shifts downstream, i.e., to the right for Ha up to 6000, and the region of separation becomes shallow. The overall separation length is about $2.53 \mathrm{~m}$ for the non-magnetic case, $1.8 \mathrm{~m}$ for $\mathrm{Ha}=3000$ and $0.13 \mathrm{~m}$ for $\mathrm{Ha}=6000$.

A Hartmann number of 6000 almost eliminates flow separation and beyond Hartmann number of 6000 , the flow is observed completely attached as can be noted from the positive skin friction values. Also it is interesting to note that the FPBL skin friction distribution lies partly between the

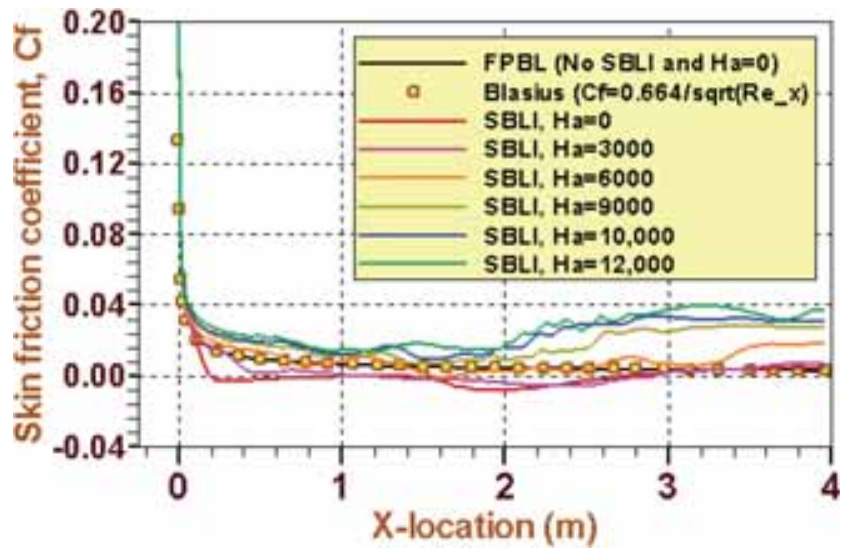

Figure 18. Skin friction distribution for various Hartmann numbers.

$\mathrm{Ha}=6000$ and $\mathrm{Ha}=9000$ cases. Though the flow separation is eliminated completely for Ha just over 6000 and beyond, the magnitude of positive values of $c_{f}$ distribution is incremented substantially when compared with the skin friction of the attached part of the separated flow cases.

\subsection{Skin friction drag with and without imposed magnetic field}

An important observation concerning the skin friction distribution is that the overall skin friction drag increases with increase in Hartmann number due to flattening of the velocity profile near the wall, resulting in large velocity gradient near wall and hence higher wall shear stresses. A plot of overall skin friction drag normalized with skin friction drag due to the non-magnetic case $(\mathrm{Ha}=0)$ is presented in figure 19 for the three grids.

For $\mathrm{Ha}=0$, the normalized skin friction drag is 1.0 and as the Hartmann number is increased, the skin friction drag increases non-linearly and reaches a value of about 31 for a Hartmann number of 12,000 in case of the coarse grid. The value of drag is 44 in medium grid and 48 in fine grid and the fine grid data are considered here for further discussions.

Since the lowest Hartmann number beyond which a complete mitigation of flow separation takes place lies between 6000 and 9000, the skin friction drag shall be considered to increase by a factor of 20 to about 35. Also, since the overall skin friction drag increases with increase in Hartmann number, it is necessary to study systematically whether the beneficial effects of MHD flow control are annulled by the high skin friction drag it brings along.

\section{Conclusions}

A proof of concept numerical investigation has been carried out for mitigation of laminar flow separation over a flat plate due to shock boundary layer interaction using 


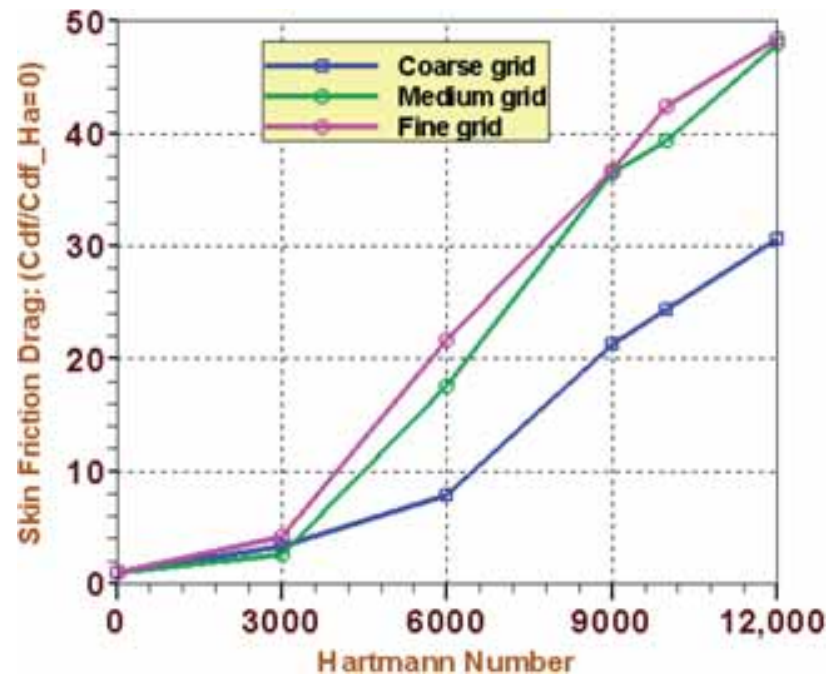

Figure 19. Normalized skin friction drag vs. Hartmann number.

magnetohydrodynamic flow control. Applying a uniform magnetic field with a Hartmann number variation from 0 to 12,000 , the flow separation has been suppressed for Ha beyond 6000 due to the Hartmann effect, which accelerates the near-wall flow. However, the overall magnitude of skin friction distribution increases drastically, resulting in large increase in skin friction drag, by as much as 20 fold as compared with the non-magnetic case, and is a cause of concern.

A future course of study shall be to ascertain whether the beneficial effect of maintaining clean unseparated flow, using MHD flow control, is offset by the marked increase in skin friction drag. Another interesting study would be to determine the effect of MHD flow control on the overall forces, including the thermodynamic and magnetic pressure forces, for a flight vehicle configuration.

\section{Acknowledgements}

The authors express their sincere gratitude to the Director, DRDL, for his constant support and encouragement.

\section{Nomenclature}

$\begin{array}{ll}A & \text { area of surface } \\ \boldsymbol{B} & \text { magnetic field vector } \\ \boldsymbol{c} & \text { characteristic speed } \\ e & \text { specific energy } \\ \boldsymbol{F}_{\boldsymbol{j}} & \text { jth component of flux vector } \\ M & \text { mach number } \\ \hat{n}_{j} & \text { jth component of surface outward normal } \\ p, p_{t} & \text { static and total pressure } \\ t & \text { time in seconds } \\ S & \text { source term vector }\end{array}$

$T \quad$ fluid temperature

$\boldsymbol{U}$ conserved variable vector

$U_{n} \quad$ contra-variant velocity

$\boldsymbol{u} \quad$ velocity vector

$V \quad$ volume of cell element

$u, v, w$ velocity components along $x, y, z$ directions

$x, y, z \quad$ Cartesian coordinate directions

\section{Greek symbols}

$\gamma \quad$ ratio of specific heats $=1.4$

$\lambda$ eigenvalue of the MHD Jacobian

$\mu_{o} \quad$ magnetic permeability of free space $\left(4 \pi \times 10^{-7}\right.$ $\mathrm{N} / \mathrm{A}^{2}$ )

$\mu_{\infty} \quad$ freestream molecular viscosity

$\rho \quad$ density of fluid

$\sigma_{e} \quad$ freestream electrical conductivity

\section{Subscripts and superscripts}

$\begin{array}{ll}(i),(v) & \text { inviscid, viscous } \\ B, m & \text { magnetic } \\ e & \text { electrical } \\ i, j & \text { vector and tensor indices } \\ n & \text { normal or contra-variant } \\ t & \text { total } \\ \infty & \text { freestream } \\ s, A, f, d, e & \text { (waves) slow, Alfven, fast, divergence, } \\ & \text { entropy }\end{array}$

\section{References}

[1] Greenblatt D and Wygnanski I 2009, Physical concepts underlying the development and application of active flow control. In: Joshlin R D and Miller D N (Eds.) Fundamentals and applications of modern flow control. Progress in Astronautics Aeronautics, vol. 231, Reston, Virginia: AIAA Inc., pp. 21-57 (Chapter 2)

[2] Kato H., Tannehill J C, Ramesh M D and Mehta U B 2002 Computation of magnetohydrodynamic flows using an iterative PNS algorithm. AIAA Paper 2002-0202

[3] Powell K G 1994 An approximate Riemann solver for magnetohydrodynamics (that works in more than one dimension). ICASE Tech. Rep. 94-24

[4] Balasubramanian R and Anandhanarayanan K 2008 Viscous computations for complex flight vehicles using CERANS with wall function. CFD J. 16(4): 386-390

[5] Balasubramanian R and Anandhanarayanan K 2014 An ideal magnetohydrodynamics solver for high speed flows. In: Proceedings of the 16th Annual CFD Symposium CP-05, NAL, Bangalore 
[6] Han S H, Lee J I and Kim K H 2009 High-order computations of MHD applications using 5th order MLP with AUSMPW+. AIAA Paper 2009-458

[7] Xisto C M, Pascoa J C and Oliveira P J 2013 A pressure-based method with AUSM-type fluxes for MHD flows at arbitrary Mach numbers. Int. J. Numer. Methods Fluids 72: 1165-1182

[8] Barth T J and Jespersen D C 1989 The design and application of upwind schemes on unstructured meshes. AIAA Paper 89-0366
[9] Ekaterinaris J 2009 Numerical investigation of the effect of magnetic fields on shock/boundary layer interaction. AIAA Paper 2009-4151

[10] Ekaterinaris J 2010 High-order numerical method for magnetohydrodynamic control of shock-induced separation. AIAA J. 48(12): 2781-2792

[11] Su W Y, Chang X Y and Zhang K Y 2010 Effects of magnetohydrodynamic interaction- zone position on shock-wave/ boundary-layer interaction. J. Propul. Power 26(5): 1053-1058 